\title{
El Derecho en la prensa escrita. Una propuesta metodológica para el aprendizaje ${ }^{1}$
}

\author{
Catalina Pons-Estel Tugores. Profesora Contratada Doctora de Derecho Civil \\ Universidad de las Islas Baleares \\ E-mail: catalina.pons-estel@uib.es \\ Marcos González Sánchez. Profesor Titular de Derecho Eclesiástico del Estado \\ Universidad Autónoma de Madrid \\ E-mail: marcos.gonzalez@uam.es
}

\begin{abstract}
In this project, the Law students are assigned the task of creating files from newspaper articles related to the subjects of study of the Degree in Law. To this end, they have selected the journalistic information and analyzed and systematized it, completing it with the most recent national and international jurisprudence. Thus, we have managed to ensure that they have a thorough knowledge of the studied topics. Furthermore, this project provides us with a very useful written material to support our classes.
\end{abstract}

Keywords: press, law, data sheet, methodology, learning, coordination, family.

\begin{abstract}
Resumen
En este proyecto los alumnos han realizado unas fichas a partir de las noticias que trataban sobre las materias objeto de estudio del Grado en Derecho publicadas en la prensa escrita. Para ello han enmarcado jurídicamente la información periodística y la han analizado y sistematizado, completándola con la jurisprudencia nacional e internacional más reciente. De este modo, hemos conseguido que el alumnado domine a fondo los temas trabajados y, además, tenemos un material escrito muy útil que nos sirve de apoyo en las clases.
\end{abstract}

\footnotetext{
${ }^{1}$ Comunicación realizada en el marco del Proyecto de Innovación Docente "El Derecho en la prensa escrita" (PID 181914) perteneciente a los proyectos de innovación y mejora de la calidad docente de la Universidad de las Islas Baleares (2018-2019), siendo la investigadora principal la Dra. Catalina Pons-Estel Tugores y formando parte del Proyecto el Dr. Marcos González Sánchez, el Dr. Pedro A. Munar Bernat, el Dr. Salvador Pérez Álvarez, la Dra. Francisca Ma Rosselló Rubert y la Dra. Beatriz Verdera Izquierdo.
} 
Palabras clave: prensa, derecho, ficha, metodología, aprendizaje, coordinación, familia.

\section{Introducción}

Muchas personas acceden al mundo del Derecho a través de la prensa escrita, ya sea en papel o en formato digital. De hecho, la utilización en clase de las noticias publicadas a diario en la prensa es un recurso muy socorrido entre el profesorado, pues es una forma de "enganchar" y motivar al alumnado. Además, la configuración actual de los Planes de Estudios exige una innovación continua en la metodología docente que se aplica en nuestras aulas. No solo los profesores, sino también los alumnos y la sociedad en general, demandamos unas mejoras y evolución constantes en la manera de ayudar al aprendizaje. Por ello, la propuesta que presentamos se basa en las siguientes claves: a) Cuando el contenido de las noticias es jurídico y afecta -o puede afectar- a la vida de las personas, resulta una materia especialmente sensible y requiere de investigaciones serias, objetivas y veraces. En especial, nos ha interesado la prensa relacionada con noticias de los tribunales de justicia (los juicios paralelos que se producen, el análisis de los límites a la libertad de expresión y de información, el lenguaje jurídico utilizado); b) Se han impulsado las metodologías activas de aprendizaje ya que hemos usado la pedagogía inversa. Así, nuestros alumnos han sido quienes han asumido la elaboración del material que en un primer momento se ha recopilado, para luego sistematizarlo y analizarlo. El objeto ha sido conseguir un material escrito muy útil que sirva de apoyo práctico en la explicación de las distintas temáticas que se abordan en las asignaturas del Grado en Derecho. La intención es que este material se publique y que sirva de apoyo en las clases; c) Se ha desarrollado el uso de herramientas TIC; y, d) El proyecto ha sido claramente interdisciplinar, ya que han colaborado profesores de tres Áreas de conocimiento, que han trabajado en equipo coordinando su labor docente.

\section{Contexto en el que se ha desarrollado el proyecto}

El equipo que ha desarrollado la experiencia docente que detallamos está integrado por profesores de Derecho Civil, Derecho Eclesiástico del Estado y Derecho Mercantil de tres Universidades distintas (Universidad de las Islas Baleares, Universidad Autónoma de Madrid y Universidad Nacional de Educación a Distancia), que imparten diversas asignaturas de sus disciplinas. Se ha trabajado coordinadamente logrando una comparativa de los diversos grupos y del aprendizaje conseguido por los alumnos.

En la Universidad de las Islas Baleares el proyecto se ha desarrollado en un grupo de Persona y Familia ( $2^{\circ}$ de Grado en Derecho) con 86 alumnos matriculados impartido por la Dra. Beatriz Verdera Izquierdo, profesora de Derecho Civil; un grupo de Derecho Eclesiástico del Estado (asignatura optativa de $4^{\circ}$ curso) con 50 alumnos matriculados, impartido por la Dra. Catalina Pons-Estel Tugores; tres grupos de Donaciones y Sucesiones ( $4^{\circ}$ de Grado en Derecho) con 60, 45 y 67 alumnos matriculados, impartidos por los 
Doctores Pedro A. Munar Bernat y Catalina Pons-Estel, profesores de Derecho Civil; un grupo de Persona y Familia ( $2^{\circ}$ de Grado en Administración y Dirección de Empresas y Derecho) con 45 alumnos matriculados impartido por la profesora Verdera y un grupo de Nociones básicas de Derecho ( $1^{\circ}$ de Grado en Turismo) con 40 alumnos matriculados impartido por la Dra. Francisca $\mathrm{M}^{\mathrm{a}}$ Rosselló Rubert, Ayudante de Derecho Mercantil. En la Universidad Autónoma de Madrid se ha trabajado con 5 alumnos de Trabajo Fin de Grado tutorizados por el Dr. Marcos González Sánchez, Profesor Titular de Derecho Eclesiástico del Estado. Por su parte, en la Universidad Nacional de Educación a Distancia se ha trabajado con el Dr. Salvador Pérez Álvarez, Profesor Titular de Derecho Eclesiástico del Estado y organizador de un Seminario celebrado en su Universidad con la finalidad de compartir y transferir los resultados logrados con el Proyecto.

Los conocimientos en materia de Persona y Familia, Donaciones y Sucesiones son muy distintos en un grupo o en otro, debido -fundamentalmente- al curso concreto que están estudiando los alumnos. Precisamente por eso, el estudio de estas materias se ha realizado de forma progresiva, según los conocimientos jurídicos del alumnado. Ello ha supuesto que los resultados alcanzados han sido diversos dependiendo del curso académico; pero, en todos ellos se ha pretendido fomentar el razonamiento lógico y el espíritu crítico.

Persona y Familia es una asignatura que, por un lado introduce en el estudio del Derecho de la Persona (personalidad jurídica, capacidad natural, capacidad de obrar, modificaciones judiciales de la capacidad de obrar, representación, etc.); y, por otro, aborda el Derecho de Familia (instituciones jurídicas que regulan las relaciones personales y patrimoniales entre los miembros de la familia y en sus relaciones con terceros). Precisamente el concepto de Familia ha sufrido una auténtica revolución en los últimos tiempos y en ella nos encontramos con familias "tradicionales", monoparentales, uniones heterosexuales u homosexuales (inscritas y no inscritas), familias reconstituidas, familias ocasionales, etc. Además, también a día de hoy hay instituciones que están viviendo una etapa crítica a la espera de una nueva regulación, como es el caso de la filiación y la dificultad que conlleva en ocasiones cohonestar la realidad biológica y la realidad jurídica de la misma.

El Derecho Eclesiástico del Estado es una rama especializada del ordenamiento jurídico que se ocupa de cómo el Derecho del Estado regula la exteriorización del fenómeno religioso en la sociedad (tanto en su dimensión individual como en la colectiva). También se pretende la familiarización del alumnado con las fuentes, los principios informadores, la legislación específica y la jurisprudencia propia del Derecho Eclesiástico, con el objetivo de poder profundizar en aquellas cuestiones que resulten de interés, especialmente interrelacionando los conocimientos de esta materia con el resto de las del ordenamiento jurídico.

Para ello, los contenidos temáticos que se trabajan son: a) concepto, evolución histórica y fuentes del Derecho Eclesiástico; b) concepto, contenido, límites, titularidad y protección jurídica del derecho fundamental de libertad religiosa; c) la dimensión individual del derecho de libertad religiosa (enseñanza, asistencia religiosa, objeción de conciencia, matrimonio canónico y acatólico -junto con su eficacia civil-); y, d) la dimensión colectiva del derecho de libertad religiosa (personalidad jurídica de las entidades religiosas, 
patrimonio cultural de las confesiones religiosas, financiación y régimen fiscal de las confesiones religiosas, etc.).

La asignatura Donaciones y Sucesiones se imparte en cuarto curso del grado en Derecho y en ella se analizan instituciones de gran arraigo social como son las derivadas del Derecho sucesorio, que llevan a concretar el destino y consecuencias del patrimonio de una persona después de su fallecimiento. En dicha asignatura se estudian distintas instituciones como pueden ser el título sucesorio, el concepto mismo de herencia y el estudio de todo el proceso que lleva a la adquisición de la misma. Y, respecto al Derecho de Donaciones se trata de concretar el procedimiento a seguir para determinar la transmisión voluntaria de bienes sin que medie un precio. Por tanto, se estudian las donaciones remuneratorias, modales, las mortis causa, con cláusula de reversión, las liberalidades de uso, etc.

Por su parte, Nociones básicas de Derecho es una asignatura de formación básica cuya función es ofrecer una panorámica de la regulación jurídica de la vida cotidiana. Sirve para tener una visión previa y general de todo aquello que, a lo largo de la carrera, se estudiará con mayor profundidad. Fundamentalmente pretende que se conozcan y comprendan -en un nivel básico- las principales nociones, instituciones y principios jurídicos.

Los Trabajos Fin de Grado son una materia globalizadora que pretende la evaluación integrada de las competencias específicas y transversales del Grado en Derecho. El objetivo de esta asignatura es conseguir que los alumnos sean aptos para realizar un trabajo de forma autónoma e individual, aplicando e integrando las competencias adquiridas durante la carrera.

\section{Objetivos}

El objetivo principal de este proyecto es valorar (a través del estudio y del comentario) la aportación de la prensa escrita al análisis del Derecho (teniendo en cuenta la repercusión que tiene en la sociedad). La prensa en papel y digital proporciona abundante información a diario de contenido jurídico, por lo que nuestra labor es determinar su grado de rigor y cientificidad. Los alumnos tendrán que enmarcar jurídicamente la información periodística y analizarla haciendo uso de las sentencias más recientes dictadas por los tribunales de justicia sobre la cuestión (tanto nacionales como del Tribunal de Justicia de la Unión Europea o del Tribunal Europeo de Derechos Humanos). En otro orden de cosas, sabemos por experiencia propia que la mejor forma de estudiar cualquier temática es teniéndola que explicar y servirse de ejemplos reales. Por eso estábamos convencidos que pidiendo a nuestros estudiantes que preparasen este material didáctico sobre cuestiones concretas, serviría de apoyo a los compañeros para conocer a fondo la temática a estudiar. Además, este material podrá seguir utilizándose en cursos futuros pues la intención es que estas herramientas tengan perdurabilidad y puedan seguir usándose en próximos cursos académicos.

Por otro lado, al ser los propios alumnos los que han expuesto los comentarios a las noticias, el resto de compañeros muestran una mejor predisposición a escuchar y aprender. 
Incluso se aprecia mucha más curiosidad y participación activa. El objetivo secundario es elaborar un material de apoyo didáctico, como ya se ha comentado, que quedará a disposición del alumnado a través de Campus Digital. Y, de cara al siguiente año académico 2019-2020, tenemos intención de publicar este material en formato libro. Consideramos que con las distintas actividades que venimos planteando durante el curso 2018-2019, trabajamos las distintas competencias propias del Grado en Derecho.

\section{Desarrollo de la innovación}

\subsection{Metodología}

Nuestro planteamiento metodológico consta de varias fases que, cronológicamente, han seguido el siguiente desarrollo: A) Elaboramos un modelo de ficha ${ }^{2}$-común para todos los alumnos- disponible online, en la página de nuestras asignaturas en Campus Digital, a fin de que diariamente se hiciera una recopilación de todas las noticias aparecidas en la prensa escrita (tanto en formato papel como digital) y se fuesen trabajando los distintos parámetros incorporados a la ficha; B) Abordamos los distintos temas en clase, con todo el grupo, mediante el sistema tradicional de clase magistral; C) Propusimos unas palabras clave e ideas básicas de cada tema; D) Formulamos preguntas relacionadas con la temática trabajada que activaron el espíritu crítico de los alumnos; E) Realizamos prácticas relacionadas con las materias trabajadas; F) Planteamos foros de debate; G) Seleccionamos las noticias que nos parecieron más interesantes (siempre desde el punto de vista jurídico) y, a través de Seminarios temáticos, trabajamos con los alumnos en la elaboración de la ficha sobre el caso asignado; H) Subimos este material didáctico a la plataforma, a fin de que todos puedan disponer de él; I) Realizamos una Mesa redonda -abierta al público en general y así siempre resulta más enriquecedora- sobre las cuestiones más candentes que habíamos trabajado, y lo hicimos mediante exposiciones breves (15 minutos aprox.) a fin de potenciar el debate ulterior entre todos los asistentes. Los alumnos están muy formados acerca de las cuestiones que se expusieron y lo cierto es que disfrutaron de participar. A lo largo de todo el proceso, los alumnos han contado con unas tutorías específicas para hacer un seguimiento de su proceso de aprendizaje, así como para orientarles en todo aquello que necesitasen; J) Además, durante el curso 2019-2020 tenemos intención de continuar con esta labor, a través de la sistematización de todo el material y la elaboración de un libro.

\subsection{Plan de trabajo}

El plan de trabajo en el que han participado todos los profesores implicados en el proyecto ha sido el siguiente:

a) Selección, bajo la dirección de su tutor, de 25 noticias sobre derecho a contraer matrimonio, adopción, custodia y ajuste a derecho de las sentencias de nulidad matrimonial

\footnotetext{
${ }^{2}$ Vid. 4.2 de esta Comunicación.
} 
canónica y posterior elaboración de una ficha de cada una de ellas por parte de los alumnos de TFG de la Universidad Autónoma de Madrid. Posteriormente, este material se transfirió a los alumnos de la Universidad de las Islas Baleares de los grupos implicados en el proyecto que nos ocupa.

b) Explicación por parte de los profesores al alumnado de la Universidad balear de la trascendencia e influencia de la prensa escrita en la sociedad, haciendo especial hincapié en la materia específica de cada docente (persona y familia, derecho de libertad religiosa, etc.).

c) Elaboración por parte del profesorado de un dossier con material de apoyo para el alumnado (artículos doctrinales, sentencias, etc.).

d) Elaboración de la propuesta de los trabajos y actividades a realizar por los alumnos. En concreto, se les pidió lo siguiente:

$1^{\circ}$ Escoger un mínimo de dos noticias publicadas en la prensa escrita (tanto en formato papel como en formato digital) que tratasen sobre dos temas distintos del derecho fundamental de libertad religiosa o de familia y sucesiones.

$2^{\circ}$ Elaborar una ficha para cada una de las noticias.

$3^{\circ}$ La extensión de cada ficha tendría que ser entre 1 (mínimo) y 3 páginas (máximo). El tipo de letra: Times New Roman; el tamaño, 12; el interlineado, sencillo.

$4^{\circ}$ En cada ficha (por este orden) tenía que aparecer:

- Tema de la noticia.

- Lugar de publicación de la noticia (la referencia completa, si es en formato papel; o el enlace completo, si es en formato digital).

- Resumen de la noticia, cuya extensión tenía que ser entre 5-10 líneas.

- En el caso de que los alumnos vieran que más medios de comunicación escrita también habían tratado esa noticia, tenían que facilitar las referencias completas.

- Un análisis comparativo o valoración del tratamiento que hacen los distintos medios de la prensa escrita acerca de una misma noticia. Esto solo era posible en el supuesto de que, efectivamente, la noticia se hubiera publicado en más de un medio.

- Indicación de cuál es el encaje normativo de la materia objeto de la noticia en nuestro país. Siempre que fuera posible, había que completarlo haciendo una comparativa con otros países,

- Cuál había sido el tratamiento jurisprudencial de esta temática en nuestro país (indicando, por supuesto, las sentencias de referencia) y en el Tribunal de Estrasburgo (indicando también cuáles son las sentencias de referencia). En la medida en que se pudiera, había que completarlo haciendo una comparativa con otros países. 
- Determinación de si se había producido algún tipo de conflicto entre libertad de expresión y libertad religiosa, en el tratamiento que se había hecho de esa noticia.

- En penúltimo lugar, presentación de unas conclusiones o valoraciones, a nivel jurídico. Aquí también podía hacerse alguna propuesta de lege ferenda.

- Finalmente había que contestar a la siguientes cuestión: ¿cuál ha sido el papel que ha jugado la prensa escrita en este caso concreto?

$5^{\circ}$ Entrega de las fichas (acompañadas de las dos correspondientes noticias).

e) Puesta a disposición del alumnado de la Universidad de las Islas Baleares del material proporcionado por los alumnos de TFG de la Universidad Autónoma de Madrid, así como del dossier con material y la propuesta de trabajos y actividades a realizar.

f) Estudio por parte de los alumnos del material recibido y selección de sus fichas.

g) Elaboración por parte de los estudiantes de un borrador de trabajo.

h) Realización de tutorías individualizadas para evaluar la viabilidad de las propuestas de los alumnos y para guiar la realización de los trabajos-fichas hasta su culminación.

j) Acceso a todo el alumnado implicado en el proyecto a las fichas realizadas.

k) Valoración en clase de las actividades desarrolladas.

\section{Resultados}

En primer lugar, hemos realizado una Mesa redonda abierta al público en general y, con ello, además de conseguir una actividad más plural, transferimos conocimientos a la sociedad en general. Además, el material didáctico elaborado no solo estará disponible para los alumnos matriculados durante el curso 2018-2019, sino que tiene un carácter de permanencia temporal, puesto que nuestra intención es que dure varios años. E incluso aspiramos a finalizar nuestra labor con la publicación -durante otro curso académico- de este material, pues es un extraordinario recurso de apoyo práctico a las materias trabajadas.

En cuanto a la evaluación, con el sistema que proponemos tendremos muchos elementos de juicio para afinar al máximo a la hora de evaluar el trabajo de cada estudiante. Así, calificaremos: 1. Las prácticas realizadas en clase; 2. La participación en los foros propuestos; 3. La elaboración de los materiales didácticos; 4. La participación en los debates (de clase y de la Mesa redonda). A su vez, hemos pedido a los alumnos que nos hicieran un breve comentario (entre 0.5 y 1 página) valorativo acerca de la metodología seguida para estudiar las líneas maestras del papel que juega el mundo jurídico en el día a día de todos los ciudadanos, a partir del tamiz de la prensa escrita. Hemos solicitado a los alumnos que nos indiquen qué les ha parecido nuestro Proyecto de innovación docente y la forma en que lo hemos llevado a cabo. Pensamos que, de este modo, conocemos mejor los pros y los contras de nuestra propuesta e iremos mejorando la forma de dar clases, ya que este es, en el fondo, el gran objetivo perseguido: lograr ser mejor docente, a fin de que los 
alumnos cada día estén más motivados para aprender y salgan mejor preparados de nuestras aulas para entrar en el mundo de la profesionalización.

Como docentes pretendemos que nuestros alumnos adquieran las competencias generales, básicas y específicas propias del Grado en Derecho. Fundamentalmente queremos contribuir a su formación integral como juristas y a posibilitarles que en el futuro gocen de total autonomía, de forma que sean capaces de enfrentarse a nuevos problemas y sepan darles solución. Para ello es básico que puedan conseguir información jurídica (ya sea Derecho positivo, jurisprudencia, doctrina), a través de las fuentes instrumentales, incluidas las digitales. Con nuestra propuesta les hemos ayudado a analizar y sistematizar material proveniente de un elemento no jurídico pero con trascendencia jurídica para la sociedad. Hemos llevado a cabo una labor de complexión -aportando el marco normativo y jurisprudencial correspondiente- a la información de la prensa escrita, dotándola de la precisión y rigurosidad científica que se le exigen. Con todo esto, no solo hemos pretendido resolver y estudiar los conflictos actuales, sino también prever y analizar las potenciales consecuencias jurídicas de la ejecución de un determinado acto. La idea de que fueran los propios alumnos quienes elaboraran los recursos didácticos nace de la convicción de los miembros de este Proyecto, de la importancia capital de la comunicación. Así, hay que saber exponer ideas, propuestas, soluciones jurídicas y hacerlo de forma ordenada, precisa, concisa -oralmente o por escrito- adaptando nuestro discurso a las circunstancias. Pensamos que la preparación y exposición por parte de los alumnos de cuestiones muy concretas, no solo apoya estas competencias sino que además, potencia la actitud crítica, el ser capaz de contrastar cualquier información jurídica analizando todas las consecuencias (políticas, económicas y sociales). Por cuanto venimos indicando, este proyecto ha promovido especialmente la metodología activa de aprendizaje; la coordinación entre diversas materias de un mismo Grado; el uso de herramientas TIC en la metodología de enseñanza-aprendizaje; la evaluación de la calidad docente y de los procesos de evaluación y aprendizaje de los alumnos; la orientación tutorial; la realización de actividades de formación complementaria; e incluso el uso de lenguas extranjeras, por cuanto las resoluciones del máximo órgano judicial europeo (el Tribunal Europeo de Derechos Humanos) que hemos utilizado están en inglés y francés; y, por último, la promoción de la elaboración de material didáctico.

Como resultado final, hemos conseguido más de 500 fichas que suponen un material valiosísimo no solo para los alumnos que han participado en este proyecto, sino también para las futuras promociones que podrán beneficiarse de este gran trabajo. Además, la aceptación por los propios alumnos participantes en este proyecto ha sido muy satisfactoria, por cuanto el $79 \%$ de ellos así lo han manifestado en una encuesta realizada de forma anónima.

\section{Conclusiones}

Tras la finalización de las distintas actividades llevadas a cabo en el marco del proyecto de innovación que ahora presentamos, los profesores integrantes del mismo nos reunimos para 
hacer una valoración del mismo y, muy brevemente, podemos reseñar las siguientes conclusiones:

a) Esta iniciativa ha resultado muy enriquecedora para los alumnos, fundamentalmente por dos motivos: porque ha supuesto una toma de contacto con compañeros de otra Universidad y porque ha posibilitado una relación de trabajo y complemento entre alumnos de distintos cursos.

b) La elaboración del material-ficha ha sido muy motivadora porque ha supuesto que los alumnos hayan elaborado un trabajo de búsqueda, estudio, sistematización y valoración crítica muy serio y riguroso, a partir de un medio de comunicación social no jurídico.

c) El hecho de que los alumnos hayan tenido que elaborar material didáctico útil para sus compañeros les ha obligado a tener que estudiar y comprender en profundidad una temática que, de entrada, es muy compleja. En cambio, se han metido tanto en su papel de "docentes" que ni siquiera se han percatado de la cantidad de horas que han invertido en la consecución del objetivo final que era el material-ficha.

d) La multidisciplinariedad del proyecto por la participación en el mismo de profesores de tres áreas de conocimiento distintas ha proporcionado una riqueza complementaria durante todo el proceso de trabajo y, sin duda, también en el resultado final. El intercambio de opiniones entre profesionales que se centran en aspectos distintos, hace que tengamos una perspectiva más amplia.

e) De entrada, más de trescientos alumnos han participado en la elaboración de este material-ficha y, por tanto, se benefician del mismo de forma directa. Pero este trabajo va más allá, pues estos recursos serán utilizados durante los próximos cursos como material de apoyo. Por esto, resulta difícil cuantificar el número total de alumnos que de forma más o menos inmediata podrán usar estos recursos. Lo que sí tenemos claro es que se tratará de un número significativamente relevante.

f) Para los profesores implicados en el proyecto esta iniciativa ha supuesto mucho trabajo extra. No obstante, también nosotros nos hemos sentido muy motivados al ver a los alumnos tan implicados en el mismo y con tantas ansias de hacer las cosas bien. Quizá esto no se ha plasmado de forma inmediata en un porcentaje mayor de alumnos aprobados (solo un $1.3 \%$ más que el año anterior); pero, sí que pensamos que nuestro proyecto ha influido en la calidad del trabajo de los alumnos y en su ánimo más positivo a la hora de llevarlo a cabo.

En cuanto a las conclusiones a las que hemos llegado acerca de la relación existente entre la prensa escrita y las informaciones jurídicas que en ella se publican, pueden sintetizarse del siguiente modo:

-El volumen de informaciones publicadas a diario con contenido jurídico reconducible a las materias impartidas en la carrera de Derecho es totalmente desbordante. No es posible trabajarlo todo. Por eso nos centramos en las noticias referidas al derecho fundamental de libertad religiosa, familia y sucesiones. 
-Dos tercios de esas noticias se referían al factor religioso y tan solo un tercio versaba sobre el Derecho de Familia y Sucesiones.

- Por lo que respecta al ámbito de Familia y Sucesiones, un $84 \%$ de las noticias hacía referencia al aspecto normativo (modificaciones, propuestas de lege ferenda, movilizaciones ciudadanas de protesta y reivindación, etc.) y solo un $16 \%$ era judicial.

-En cuanto a las noticias de libertad religiosa, un 67\% hacían referencia a los ataques sufridos por fieles de distintas confesiones (principalmente, católicos), a las ofensas contra los sentimientos religiosos (casi un 6\%) y el resto iba referido a la conservación del patrimonio histórico cultural de titularidad religiosa, a las inmatriculaciones a favor de la Iglesia católica, a debatir la financiación de las confesiones religiosas y a la enseñanza religiosa en los centros docentes públicos.

-Y, finalmente, lo que más ha llamado la atención de los alumnos ha sido la constatación de las marcadas cargas ideológicas de la prensa escrita, de manera que una misma noticia puede diferir en $180^{\circ}$, dependiendo de cuál sea su medio de publicación.

\section{Referencias}

AZURMEndi, A. (2016). Derecho de la comunicación. Guía jurídica para profesionales de los medios. Navarra: Ediciones Universidad de Navarra, EUNSA.

BouZa Álvarez, F. (2006). "La influencia de los medios en la formación de la opinión pública: los procesos jurídicos y los juicios paralelos" en Cuadernos de Derecho judicial, nº 16, págs. 35-60.

Carpizo, J. Los Medios de Comunicación Masiva y el Estado de Derecho, la Democracia, la Política y la Ética, en <https://revistas.juridicas.unam.mx/index.php/derechocomparado/article/view/3609/4361> [Consulta: 14 de junio de 2018]

Fayos Gardó, A. (2000). Derecho a la intimidad y medios de comunicación, Centro de Estudios Políticos y Constitucionales, Madrid.

Fuentes OsORIO, J.L. (2005). "Los medios de comunicación y el Derecho Penal”, Revista Electrónica de Ciencia Penal y Criminología, 51 págs., en <http://criminet.ugr.es/recpc/07/recpc07-16.pdf> [Consulta: 14 de octubre de 2018]

GARCía PÉREZ, C.L. (2015). "La responsabilidad civil de los medios de comunicación por vulneración del derecho al honor", Aranzadi civil-mercantil. Revista doctrinal, vol. 2, nº 1, págs. 27-44.

GutiérRez Del Moral, Ma.J. (2009). "Libertad religiosa y medios de comunicación. Derecho de acceso y protección de la libertad religiosa", Revista General de Derecho Canónico y Derecho Eclesiástico del Estado, $\mathrm{n}^{\circ} 19$.

LÓPEZ ORTEGA, J.J. (2006). "Información y Justicia: la dimensión constitucional del principio de publicidad judicial y sus limitaciones", Cuadernos de Derecho judicial, nº 16, págs. 93-136.

Monroy RodríGuez, Á. A. (2015). "Construcción del enemigo del derecho penal desde los medios de comunicación”, Advocatus, vol. 12, n²4, págs. 31-45. 
MourA, W., El Derecho a la intimidad y los medios de comunicación en <http://www.egov.ufsc.br/portal/conteudo/el-derecho-la-intimidad-y-los-medios-decomunicaci\%C3\%B3n > [Consulta: 22 de febrero de 2019]

PÁEZ, T. (2013). “Libertad de expresión, democracia y propiedad”, Derecom, nº 12, 19 págs.

Soto Navarro, S. (2005). "La influencia de los medios en la percepción social de la delincuencia", Revista Electrónica de Ciencia Penal y Criminología, 46 págs., en <http://criminet.ugr.es/recpc/07/recpc07-09.pdf> [Consulta: 17 de marzo de 2019]

-Libertad de expresión, legislación sobre medios de comunicación y difamación, en <https://www.mediadefence.org/sites/default/files/resources/files/MLDI.IPI\%20defamation\%20manu al.Spanish.pdf> [Consulta: 22 de febrero de 2019]

-Los medios de comunicación y el buen gobierno en <http://www.unesco.org/new/es/unesco/events/prizes-and-celebrations/celebrations/internationaldays/world-press-freedom-day/previous-celebrations/worldpressfreedomday200900000/theme-mediaand-good-governance/> [Consulta: 18 de diciembre de 2018] 